\title{
Kinematic wavefield attributes based multidimensional prestack data reconstruction
}

\author{
Hao Zhao*12, Volodya Hlebnikov ${ }^{1}$, Espen Harris Nilsen ${ }^{2}$, Andreas Kjelsrud Evensen ${ }^{2}$, Thomas Greiner ${ }^{12}$, \\ Jan Erik Lie ${ }^{2}$, Leiv-J. Gelius ${ }^{1}$ \\ 1. University of Oslo, Norway, ${ }^{2}$ Lundin Energy, Norway.
}

\section{Summary}

The $4 \mathrm{D} / 5 \mathrm{D}$ interpolation and regularization methods effectively improve the quality of seismic imaging. In addition to the Fourier domain interpolation method, 5D interpolation based on the common reflection surface (CRS) method has attracted more and more attention due to simplicity of its implementation and effectiveness of performance. However, the main challenge of this method is the heavy calculation in parameter estimation. To overcome this limitation, we introduce a kinematic wavefield attributes based prestack data interpolation and regularization method. This method uses gradient structure tensor (GST) and quadratic structure tensor (QST) methods to extract kinematic wavefield attributes (local slopes and curvatures) and use them for fast 3D zero-offset (ZO) CRS parameter estimation. The derived parameters are then used for 3D CRS based prestack interpolation and regularization. The proof of concept is demonstrated on datasets acquired by TopSeis. The corresponding results show that the improved efficiency of the GST/QST based method in kinematic wavefield attribute extraction and 3D ZO CRS parameter estimation. Moreover, the interpolated and regularized TopSeis prestack data derived from the subsequent 3D ZO CRS proves the simplicity and effectiveness of this method.

\section{Introduction}

The irregularity and sparsity of data sampling caused by the limitations of seismic acquisition is one of the major challenges in seismic processing and imaging. The accurate interpolation and regularization of prestack data are essential for image quality and further interpretation.

To solve this problem, many interpolation and regularization methods have been proposed. The main categories of these include methods based on Fourier transform, such as minimum weighted norm interpolation (Liu \& Sacchi 2004; Zwartjes \& Sacchi 2007), and methods based on predictive filtering (Naghizadeh \& Sacchi 2009; Liu \& Fomel 2011), which use fast Fourier transforms (FFTs) and can well handle data interpolation with regular grids. To further solve the interpolation problem in irregular grids, the anti-leakage Fourier transform reconstruction (Xu et al, 2005; Poole, 2010), projection onto convex sets (Abma \& Kabir, 2006) and rank-reduction methods (Trickett 2008; Trickettet al. 2010) were proposed and widely used in the industry. Another category of method is interpolation with wavefield attributes (Höcht et al. 2009; Baykulov \& Gajewski 2009;
Xie \& Gajewski 2017), which derives wavefield attributes from data itself and uses them for interpolation. This method is flexible in handling both regular and irregular grids, and is simple to implement, but it is limited by the high computational cost in wavefield attribute extraction. To solve this problem and make the method feasible in industrial applications, we propose a fast and robust kinematic attributes extraction method (Waldeland et al. 2018, 2019) and a 3D ZO CRS-based multidimensional prestack data reconstruction method. In the following, we introduce detailed theory and proof-of-concept numerical results.

\section{Theory and Method}

\section{D ZO CRS}

CRS operator can be formulated as a second-order Taylor expansion of the traveltime surface around the reference center point. This operator can be derived by paraxial ray theory (Schleicher et al., 1993) or by the geometrical approach of Höcht et al. (1999). The 3D ZO CRS operator is given by:

$t^{2}(\mathbf{x}, \mathbf{h})=\left(t_{0}+\mathbf{A} \Delta \mathbf{x}\right)^{2}+\mathbf{B} \Delta \mathbf{x}^{2}+\mathbf{C h}^{2}$

where $t, \mathbf{x}$ and $\mathbf{h}$ denote the two-way travel time and coordinates of the midpoint and half-offset of a selected point in the reflection surface. $t_{0}$ is the ZO two-way travel time, and $\Delta \mathbf{x}$ is the vector from the central midpoint $\left(\mathrm{x}_{0}, \mathrm{y}_{0}\right)$ to the considered midpoint:

$$
\mathbf{h}=\left[\begin{array}{l}
h_{x} \\
h_{y}
\end{array}\right], \Delta \mathbf{x}=\mathbf{x}-\mathbf{x}_{0}=\left[\begin{array}{l}
\Delta x \\
\Delta y
\end{array}\right]=\left[\begin{array}{c}
x-x_{0} \\
y-y_{0}
\end{array}\right] .
$$

where $\mathbf{A}, \mathbf{B}$ and $\mathbf{C}$ are the CRS model parameters describing 3D ZO case, which are generalized to vectors and matrices:

$$
\mathbf{A}=\left[\begin{array}{l}
\frac{\partial t}{\partial x} \\
\frac{\partial t}{\partial y}
\end{array}\right]_{\mathbf{x}=\mathbf{x}_{0}, \mathbf{h}=0}, \mathbf{B}=t_{0}\left[\left.\begin{array}{cc}
\frac{\partial^{2} t}{\partial x^{2}} & \frac{\partial^{2} t}{\partial x \partial y} \\
\frac{\partial^{2} t}{\partial x \partial y} & \frac{\partial^{2} t}{\partial y^{2}}
\end{array}\right|_{\mathbf{x}=\mathbf{x}_{0}, \mathbf{h}=0}, \mathbf{C}=\left.t_{0}\left[\begin{array}{cc}
\frac{\partial^{2} t}{\partial h_{x}^{2}} & \frac{\partial^{2} t}{\partial h_{x} \partial h_{y}} \\
\frac{\partial^{2} t}{\partial h_{x} \partial h_{y}} & \frac{\partial^{2} t}{\partial h_{y}^{2}}
\end{array}\right]\right|_{x=\mathbf{x}_{0}, \mathbf{h}=0}\right.
$$

The CRS model parameter $\mathbf{A}$ relates to the emergence angle at the measurement surface, which is expressed with the elements of first-order derivatives of CRS travel time. B and $\mathbf{C}$ are matrices composed of time-scaled second-order derivatives related to the curvatures of the normal-incidencepoint wave as well as the normal wave. 


\section{Kinematic wavefield attributes based multidimensional prestack data reconstruction}

\section{$\underline{\text { Fast and robust kinematic wavefield attribute estimation }}$}

Kinematic wavefield attribute (CRS parameter) estimation is commonly based on parameter searches that maximize the semblance (Neidell and Taner, 1971), which is a computationally expensive process. Since 3D ZO CRS has eight parameters in model parameterization, an accurate and efficient parameter estimation method is always needed in its application. Using GST/QST methods to estimate CRS parameters based on local slope and curvature (Waldeland et al., 2018, 2019) proves that the accuracy of CRS parameter estimation is comparable, and the efficiency of estimation can be greatly improved compared to pragmatic and full semblance search methods.

The proposed fast and robust kinematic wavefield attribute estimation method extracts local slopes and curvatures from 3D CMP stack using GST and QST methods. The C parameter is directly derived from the velocity guide. The $\mathbf{A}$ and $\mathbf{B}$ parameters associated with local slopes and curvatures which can be extracted from 3D CMP stack by GST/QST methods. This GST/QST-based method can derive accurate kinematic parameters for CRS applications in general. Optionally, further global optimization based on the GST/ QST method can further optimize the estimated parameters.

Kinematic wavefield attribute based Interpolation and $\underline{\text { regularization }}$

Based on the derived kinematic wavefield attritubutes, the 3D ZO CRS can be parametrized and used for the interpolation and regularization of $3 \mathrm{D}$ prestack data set. The whole process includes following steps:

1. Assume the input prestack 3D CMP gathers are sorted and binned into common (finite) offset planes based on midpoint/offset coordinates $(\mathbf{m}, \mathbf{h})$ and 3D geometry. The midpoint and offset are defined with:

$\left.\mathbf{m}=\left[\begin{array}{l}m_{x} \\ m_{y}\end{array}\right]=\frac{1}{2}\left(\begin{array}{l}s_{x} \\ s_{y}\end{array}\right]+\left[\begin{array}{l}r_{x} \\ r_{y}\end{array}\right]\right), \mathbf{h}=\left[\begin{array}{l}h_{x} \\ h_{y}\end{array}\right]=\frac{1}{2}\left(\left[\begin{array}{l}r_{x} \\ r_{y}\end{array}\right]-\left[\begin{array}{l}s_{x} \\ s_{y}\end{array}\right]\right)$,

where $\left(s_{x}, s_{y}\right)$ and $\left(r_{x}, r_{y}\right)$ denote the source and receiver coordinates.

2. Generate the 3D CMP stack based on the velocity guide, and estimate kinematic wavefield attributes and ZO CRS parameters.

3. For every 3D CMP, generate a 3D CRS super-gather based on defined midpoint aperture. The 3D CRS super-gather is generated by selecting the adjacent 3D CMPs according to the defined CRS midpoint apertures. Figure 1 illustrates a CRS super-gather and the corresponding seismic traces in 3D geometry, where the half midpoint aperture ( 2 cells) has been used for both inline and xline direction.

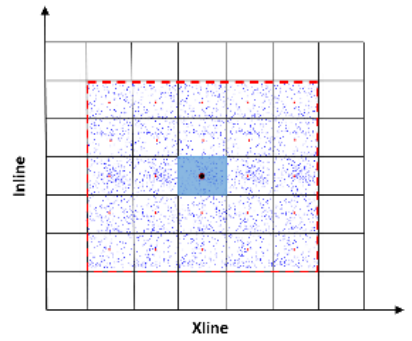

Figure 1. A selected CRS super-gather (area marked with a red dash line). The reference 3D CMP is the central bin marked with blue. The blue dots represent the sorted seismic traces in each 3D CMP. The red dots indicate the center of each bin.

4. Once the 3D CRS super-gather is generated, the traces of considered 3D CMP gather can be interpolated or regularized by employing $3 \mathrm{D}$ ZO CRS. For every trace within the considered 3D CMP, the following steps are applied for the interpolation of missing trace and/or regularization of existing trace:

- Select traces according to the defined offset aperture. The offset aperture is given by the magnitude of the offset vector $\|\Delta \mathbf{h}\|=\sqrt{\Delta h_{x}^{2}+\Delta h_{y}^{2}}$, or the number of offset planes. (Figure 2a).

- For every sample of a target finite-offset trace, samples from adjacent traces derived from 3D CRS surface are collected (Figure 2b). The ZO CRS parameters derived from step 2 and the coordinates of traces are used for the calculation of CRS traveltime surfaces. The interpolation and regularization are then applied by simple arithmetic averaging of located trace samples. (Interpolation methods based on a more accurate weighted summation are being developed).

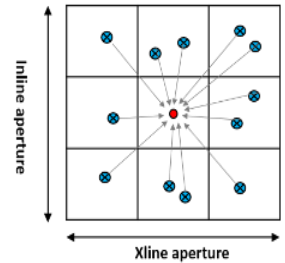

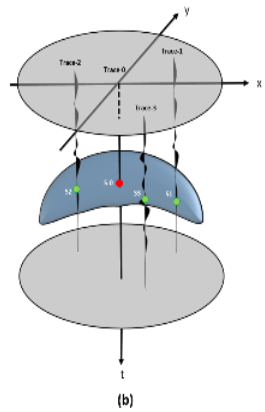

Figure 2 (a) Schematics of selected adjacent traces(blue dots) for 3D ZO CRS based interpolation/regularization of the considered finiteoffset trace (red dot). (b) Interpolation or regularization of a sample of the target finite-offset trace (red dot) by the samples of adjacent traces (green dots). The common-reflection-surface derived from $3 \mathrm{D}$ ZO CRS is represented by a blue surface. 


\section{Kinematic wavefield attributes based multidimensional prestack data reconstruction}

\section{$\underline{\text { TopSeis marine seismic data acquisition }}$}

The TopSeis acquisition technology was invented by Lundin and CGG (Vetle et al., 2017) to improve seismic imaging and inversion in shallow and intermediate targets of the Barents Sea, where the traditional seismic acquisition method suffers from the challenge of seismic imaging due to the hard and rugose water bottom and high seismic velocity of shallow geological layers.

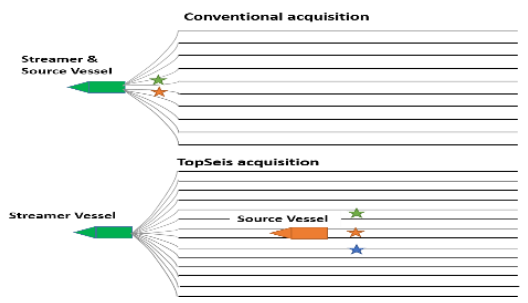

Figure 3. Conventional and TopSeis acquisition configuration

As shown in Figure 3, compared with the conventional acquisition that uses a single vessel towing two airgun seismic sources in front of arrays of multiple streamers. TopSeis acquisition utilizes dual-vessel acquisition and shooting over seismic spread strategies, which uses a separate source vessel shooting with triple or more sources in the middle of streamer spread. In such a configuration, the TopSeis acquisition demonstrates superior improvements than conventional acquisition in many aspects such as splitstep offset, increased near-offset coverage including zerooffset and improved azimuth coverage for near-offsets.

\section{Example}

In the example, we use TopSeis acquired marine data set in the Barents Sea in 2017. This TopSeis survey was operated with source vessel towing 3 airgun sources over the middle of 14 cables towed by the streamer vessel. The shot, receiver intervals are $8.33 \mathrm{~m}$ and $12.5 \mathrm{~m}$ respectively. The cable length is $7050 \mathrm{~m}$, and the spacing between the cables is $50 \mathrm{~m}$. Based on this configuration, the acquisition achieves nominal fold coverage: 140 and a 3D CMP bin size: $6.25 \times 8.333 \mathrm{~m}$.

In our test, a swath of 3D prestack data set $(2.75 \mathrm{~km}$ x $20 \mathrm{~km})$ was selected, and sorted into 140 common offset planes. The bin center of each finite-offset plane starts from $-3475 \mathrm{~m}$ to $3475 \mathrm{~m}$ with an increment of $50 \mathrm{~m}$. For the kinematic wavefield attributes based interpolation, we first extracted the kinematic wavefield attributes and ZO CRS parameters from the 3D CMP stack, and applied the ZO CRS based interpolation and regularization to the prestack data. The proposed GST/QST based kinematic wavefield attributes method proves the high efficiency in the CRS parameter estimation. The whole parameter estimation uses 50 minutes with a single thread on a CPU cluster with 32 cores and 500 GB memory. In the interpolation, the half midpoint aperture
$2 \times 2$ (inline/xlines), and adaptive half-offset aperture settings (min: 1 and max: 5 offsets) are used in the interpolation and regularization.

As shown in Figure 4, the kinematic wavefield attributes based reconstruction demonstrates promising results, and the quality of interpolation of missing traces is very good. We can also see the significant improvements on interpolated near-offset data (Figure 5). The interpolation nicely reconstructs the main reflection and diffraction in both inline and xline sections. The time-slices (Figure 6) of the nearoffset data shows the good reconstruction of missing data, further proving the superior quality of the proposed interpolation and regularization method.

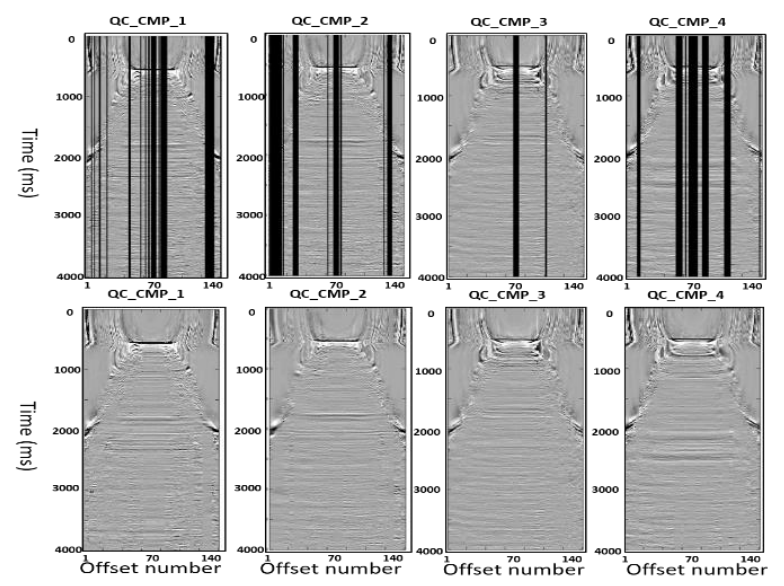

Figure 4. 3D CMP gathers before (top) and after (bottom) interpolation (moveout correction applied)
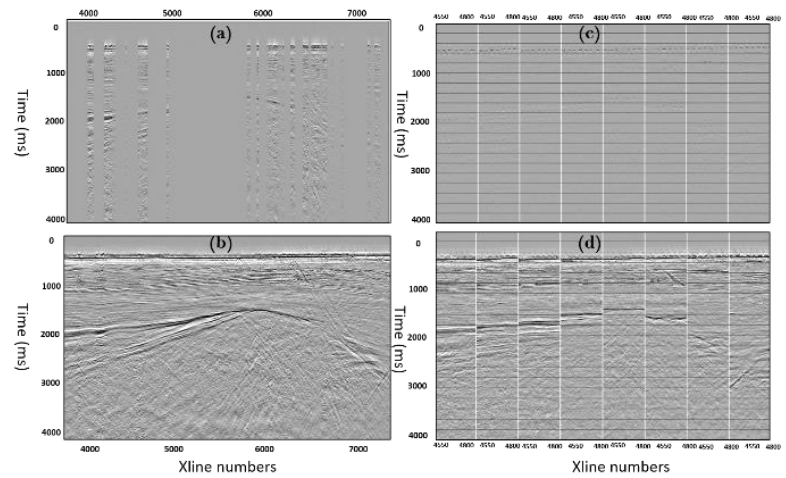

Figure 5. Comparison of raw and interpolated near-offset data (offset $25 \mathrm{~m}$ ). (a) Inline section from raw data (b) Inline section from interpolated data, (c) Xline section from raw data, and (d) the same xline section from interpolated data. 


\section{Kinematic wavefield attributes based multidimensional prestack data reconstruction}

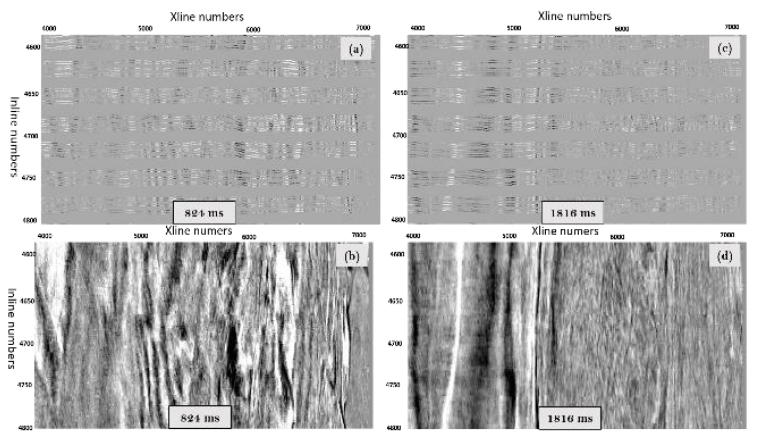

Figure 6. Time slices comparison of raw and interpolated near-offset data (offset $25 \mathrm{~m}$ ). Time slices from the raw data are shown in figures (a) and (c), the corresponding time slices from interpolated data are shown in figures $(b)$ and $(d)$.

In order to verify the quality of the interpolation and regularization, the results of the proposed method are compared with the results produced by the industrial standard anti-leakage Fourier transform reconstruction method. The two methods are applied on the same data set and the interpolated common (finite) offset datasets are migrated to generate the final migrated image. As we can see from the comparison, the migrated image (Figure 7) obtained from the proposed method shows very similar quality to the image obtained from the anti-leakage Fourier transform method. In addition, comparisons from time slices of migrated finite-offset cubes show that the migrated offset cubes are very comparable. (Figure 8 ).
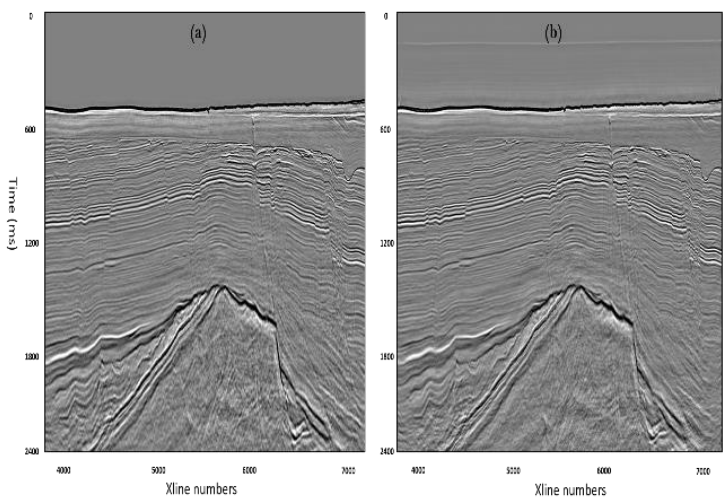

Figure 7. Comparison of migrated image from anti-leakage Fourier transform interpolated data (left) and from data prepared by kinematic wavefield attributes based prestack data reconstruction.

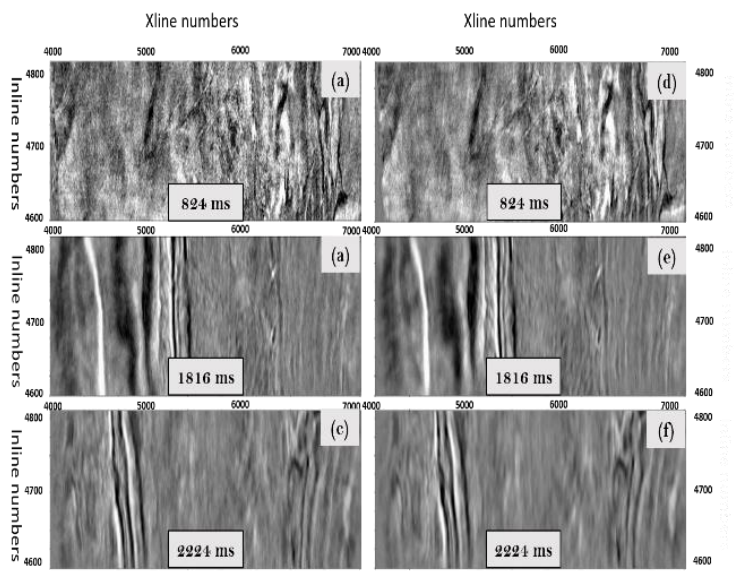

Figure 8. Comparison of time slices of migrated finite-offset cube (offset $975 \mathrm{~m}$ ). Time slices from anti-leakage Fourier transform reconstruction method show in figures (a), (b) and (c), the corresponding time slices from kinematic wavefield attributes based interpolation display at figures (d), (e) and (f).

\section{Conclusion}

In this study, we introduce the kinematic wavefield attributes based multidimensional prestack data reconstruction method. We recommend using GST/QST method for kinematic wavefield attribute extraction and fast and robust 3D ZO CRS parameter estimation. Compared with the conventional semblance based search methods, the proposed parameter estimation method has sufficient accuracy and higher efficiency.

We also demonstrate the 3D ZO CRS based interpolation and regularization on the TopSeis data set. The results show a good reconstruction of the missing prestack data. Through further comparison with the anti-leakage Fourier-transform reconstruction method, we can see that this proposed method can achieve a quality comparable to the standard industry interpolation method. In addition, due to the simplicity of its concept and implementation, we believe that the proposed method may be an effective and efficient interpolation method for prestack data interpolation and regularization.

\section{Acknowlegments}

We thank Lundin Energy for sponsoring the project and allowing the research results to be published. Hao Zhao would like also thank Odd Kolbjørnsen in Lundin Energy and Vetle Vinje, Peng Zhao in CGG for their constructive review and discussion. 


\section{REFERENCES}

Abma, R., and N. Kabir, 2006, 3D interpolation of irregular data with POCS algorithm: Geophysics, 71, no. 6, E91-E97, doi: https://doi.org/10.1190/ 1.2356088 .

Baykulov, M.,and D. Gajewski, 2009, Prestack seismic data enhancement with partial common-reflection-surface (CRS) stack: Geophysics, 74, no. 3, V49-V58, doi: https://doi.org/10.1190/1.3106182.

Höcht, G., E. D. Bazelaire, P. Majer, and P. Hubral, 1999, Seismics and optics: hyperbolae and curvatures: Geophysical Prospecting, 42, $261-281$.

Höcht, G., P. Ricarte, S. Bergler, and E. Landa, 2009, Operator oriented CRS interpolation: Geophysical Prospecting, 57, 957-979.

Liu, B., and M. D. Sacchi, 2004, Minimum weighted norm interpolation of seismic records, Geophysics, 69, 1560-1568, doi: https://doi.org/10.1190/ 1.1836829 .

Liu, Y., and S. Fomel, 2011, Seismic data interpolation beyond aliasing using regularized nonstationary autoregression, Geophysics, 76, no. 5, V69V77, doi: https://doi.org/10.1190/geo2010-0231.1.

Naghizadeh, M., and M. D. Sacchi, 2009, f-x adaptive seismic-trace interpolation: Geophysics, 74, no. 1, V9-V16, doi: https://doi.org/10.1190/1 .3008547 .

Neidell, N. S., and M. T. Taner, 1971, Semblance and other coherency measures for multichannel data: Geophysics, 36, 482-497, doi: https://doi.org/ $10.1190 / 1.1440186$.

Poole, G., 2010, 5D data reconstruction using the anti-leakage Fourier Transform: 72nd EAGE Conference, Expanded Abstracts, doi: https://doi.org/ $10.3997 / 2214-4609.201400654$

Schleicher, J., M. Tygel, and P. Hubral, 1993, Parabolic and hyperbolic paraxial two-point traveltimes in 3D media: Geophysical Prospecting, 41, 495513, doi: https://doi.org/10.1111/j.1365-2478.1993.tb00581.x.

Taner, M., and F. Koehler, 1969, Velocity spectra - Digital computer derivation and applications of velocity functions: Geophysics, 34, 859-881, doi: https://doi.org/10.1190/1.1440058.

Trickett, S., 2008, F-xy cadzow noise suppression: CSPG CSEG CWLS Convention, 303-306.

Trickett, S., L. Burroughs, A. Milton, L. Walton, and R. Dack, 2010, Rank reduction-based trace interpolation: SEG Technical Program, Expanded Abstracts, 3829-3833, doi: https://doi.org/10.1190/1.3513645.

Vinje, V., J. E. Lie, V. Danielsen, P. E. Dhelie, R. Siliqi, C.-I. Nilsen, E. Hicks, and A. Camerer, 2017, Shooting over the streamer spread: First Break, 35, 97-104.

Waldeland, A. U., T. A. Coimbra, J. H. Faccipieri, A. H. S. Solberg, and L. J. Gelius, 2019, Fast estimation of prestack common reflection surface parameters: Geophysical Prospecting, 67, 1163-1183, doi: https://doi.org/10.1111/1365-2478.12740.

Waldeland, A. U., H. Zhao, J. H. Faccipieri, A. H. Schistad Solberg, and L. J. Gelius, 2018, Fast and robust common-reflection-surface parameter estimation: Geophysics, 83, no. 1, O1-O13, doi: https://doi.org/10.1190/geo2017-0113.1.

Xie, Y. and Gajewski, D., 2017. 5-D interpolation with wavefront attributes: Geophysics Journal International, 211, 897-919, doi: https://doi.org/10 $.1093 / \mathrm{gji} / \mathrm{ggx} 334$.

Xu, S., Y. Zhang, D. Pham, and G. Lambare, 2005, Anti leakage Fourier transform for seismic data regularization: Geophysics, 70, V87-V95, doi: https://doi.org/10.1190/1.1993713.

Zwartjes, P. M., and M. D. Sacchi, 2007, Fourier reconstruction of nonuniformly sampled, aliased seismic data: Geophysics, 72, no. 1, V21-V32, doi: https://doi.org/10.1190/1.2399442. 